\title{
Feasibility of the REDCap platform for Single Center and Collaborative Multicenter CMR Research
}

\author{
Timothy C Wong ${ }^{1}$, Gaby Captur², Uma Valeti ${ }^{3}$, James Moon², Erik B Schelbert ${ }^{*}$ \\ From 17th Annual SCMR Scientific Sessions \\ New Orleans, LA, USA. 16-19 January 2014
}

\section{Background}

REDCap (Research Enterprise Data CAPture) software may provide a feasible platform for CMR Centers to: a) capture clinical throughput securely for research purposes, and 2) collaborate using a common platform for either distributed or centralized data storage. REDCap may facilitate CMR Centers' participation in the research enterprise, especially those with limited resources. REDCap may catalyze multicenter studies with "distributed data collection" where CMR sites can clone shared data dictionaries across sites for subsequent compilation into a singular master data file.

\section{Methods}

Investigators without prior REDCap training created a REDCap database hosted by the University of Pittsburgh with software developed by Vanderbilt University. In an IRB approved protocol. A full time research nurse consented patients referred for clinical CMR scans and abstracted patients' clinical data into REDCap during CMR scanning. Data elements included: key summary findings from CMR (volumes, LGE, etc) and all prior cardiac imaging data including full (unstructured) reports pasted into text fields or uploaded pdf attachments; blood testing acquired during IV placement; demographics; comorbidity; and medications. Front end quality assurance measures included data formats, data ranges, and redundant identifiers. We maximized data security by configuring users' rights, limiting data exports to the only the principal investigator, and logging every time stamped manipulation to the database. Adverse event queries occurred biannually. Data were entered via a web browser and stored on encrypted servers behind firewalls. Data were exported to statistical software packages for analysis.

\section{Results}

We established single center feasibility. We enrolled $>3000$ consecutive individuals over 3.5 years. This cohort formed the basis for several publications and ongoing investigations. No security breaches occurred. To demonstrate feasibility of multicenter data collection, we imported two entire REDCap project databases (field names and structure without data) from the University of Minnesota and the University College of London via email exchange of a .csv file. We cloned the databases rendering them operational in $<5$ minutes similar to a new collaborative project site. We also queried the world map of REDCap capable sites (http://www.project-redcap. org) to demonstrate the potential for global multicenter data collection (Figure 1).

\section{Conclusions}

REDCap is widely available and provides a robust platform for clinical CMR research. REDCap provides sites with limited resources a powerful means for rich data collection for clinical CMR research. Scarce resources can then be directed to the burden of rich data collection necessary for robust risk adjustment and multiple hypothesis testing. Shared REDCap data dictionaries are feasible and have the potential to enhance collaboration. REDCap has the potential to accelerate clinical CMR research.

\section{Funding}

American Heart Association The Pittsburgh Foundation.

${ }^{1}$ University of Pittsburgh, Pittsburgh, Pennsylvania, USA

Full list of author information is available at the end of the article 


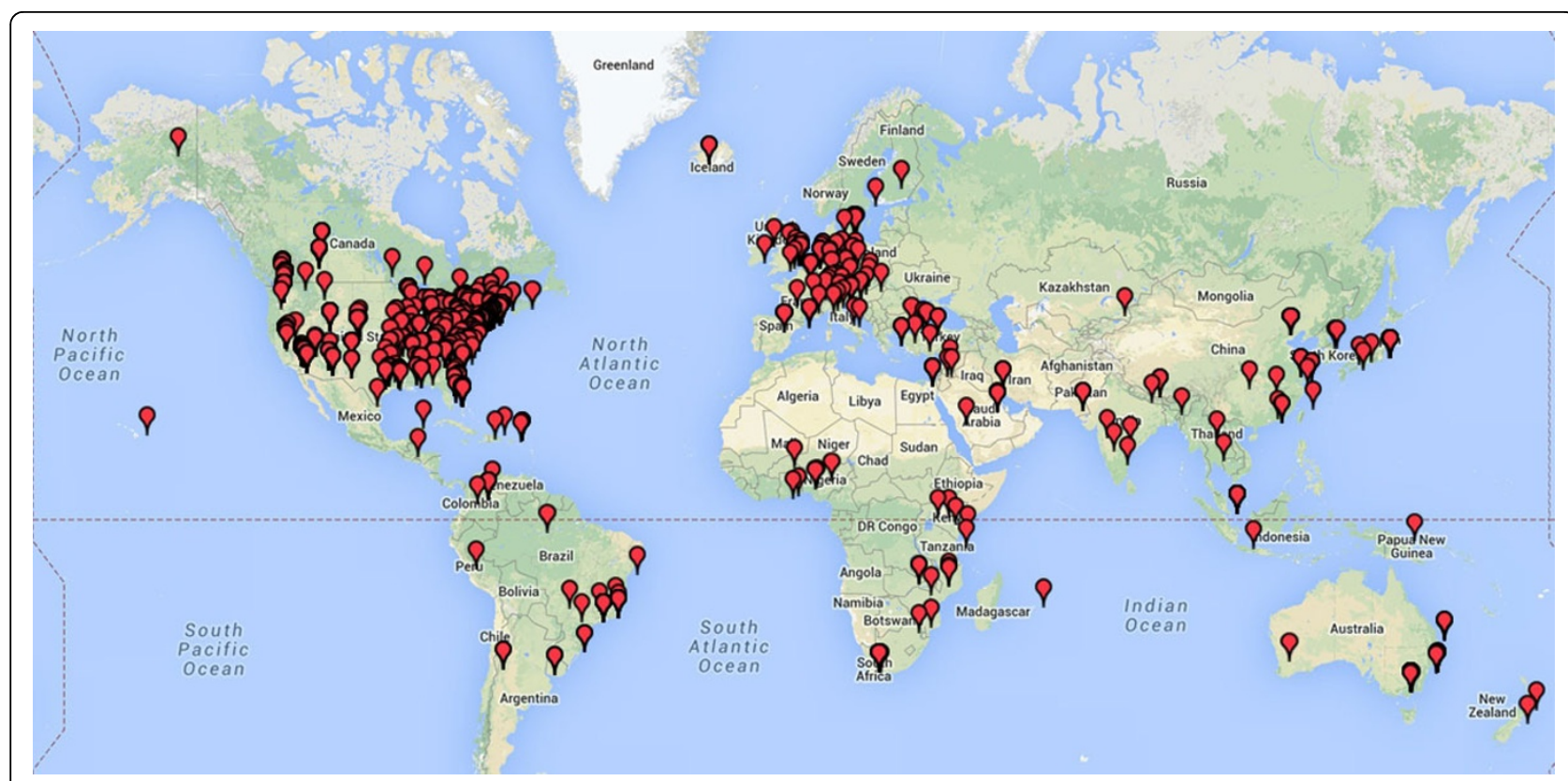

Figure 1 REDCap platforms are available worldwide and provide pre-existing infrastructure thus simplifying multicenter collaborations.

\section{Authors' details}

${ }^{1}$ University of Pittsburgh, Pittsburgh, Pennsylvania, USA. ${ }^{2}$ University College London, London, UK. ${ }^{3}$ University of Minnesota, Minneapolis, Minnesota, USA.

Published: 16 January 2014

doi:10.1186/1532-429X-16-S1-P89

Cite this article as: Wong et al: Feasibility of the REDCap platform for

Single Center and Collaborative Multicenter CMR Research. Journal of

Cardiovascular Magnetic Resonance 2014 16(Suppl 1):P89.
Submit your next manuscript to BioMed Central and take full advantage of:

- Convenient online submission

- Thorough peer review

- No space constraints or color figure charges

- Immediate publication on acceptance

- Inclusion in PubMed, CAS, Scopus and Google Scholar

- Research which is freely available for redistribution 